\title{
Research on Proximity Magnetic Field Influence in Measuring Error of Active Electronic Current Transformers
}

\author{
Weijiang $\mathrm{Wu}^{1,2}$, Yan $\mathrm{Xu}^{3}$, Shihai Yang ${ }^{1,2}$, Shufeng $\mathrm{Lu}^{1,2}$, Xia Xiao ${ }^{3}$ \\ ${ }^{1}$ Electric Power Research Institute, Jiangsu Electric Power Company, Nanjing 211103, China \\ ${ }^{2}$ Key Laboratory of Electric Energy Metering, State Grid Corporation, Nanjing 210019, China \\ ${ }^{3}$ School of Electrical and Electronic Engineering, Huazhong University of Science and Technology, Wuhan 430074, China
}

\begin{abstract}
The principles of the active electronic current transformer (ECT) are introduced, and the mechanism of how a proximity magnetic field can influence the measuring of errors is analyzed from the perspective of the sensor section of the ECT. The impacts on active ECTs created by three-phase proximity magnetic field with invariable distance and variable distance are simulated and analyzed. The theory and simulated analysis indicate that the active ECTs are sensitive to proximity magnetic field under certain conditions. According to simulated analysis, a product structural design and the location of transformers at substation sites are suggested for manufacturers and administration of power supply, respectively.
\end{abstract}

\section{Introduction}

In recent years, there has been a wave of construction of smart grids. Electronic instrument transformers, which are an indispensable part of an intelligent substation, have been developed. New types of electronic instrument transformers will be widely used in the power grid system because of their advanced sensing mechanism, simple insulation structure, immunity to magnetic saturation, wide dynamic range, excellent frequency response, and ability to adapt to developments in automatic protection [1-4].

At present, electronic current transformers (ECTs) are classified into active ECTs and passive ECTs. Active ECTs are ECTs that have a primary current sensor or primary converter with a power supply. Passive ECTs refer to ECTs having a primary current sensor or primary converter without a power supply. Active ECTs with Rogowski coil are the most practical and have been widely applied after many years development research. Rogowski coils used to be applied in high pulse current measuring and large direct current measuring [5-9]. Owing to their measuring output power being small, Rogowski coils are not able to drive metering and protection devices in traditional substations [10]. Hence, their applications in metering and protection devices at substation sites are restricted. With the rapid development of smart grid and widely launch of intelligent substations, the shift to digitization in the power system made present-day metering and protection devices not need large power drive any more. Rogowski coils applied in current measurement in intelligent substations adapt to the development tendency and they are in extensive application at present stage [11].

In analyzing tests and runtime conditions so far, active ECTs, at intervals, have shown excessive errors in installation testing at substation sites, although they have passed type tests and delivery tests. Existing research into real error characteristics under online conditions is insufficient. The disparity between the error characteristics of active ECTs in the laboratory and at the substation site severely affects metering credibility. As a consequence, it is necessary to research error characteristics of active ECTs under the two conditions to determine a congruent relationship of transformer error characteristics and to provide a rationalization proposal for the application of active ECTs.

This paper focuses on impacts created by proximity magnetic field on active ECTs. The impacts of three-phase proximity magnetic field are simulated and analyzed by magnetic field simulation software. Several suggestions are given with regard to conclusion from simulation analysis.

\section{Principle of Active Electronic Current Transformers and Sources of Error}


Principle of Rogowski Coils. Rogowski coil, based on non-magnetic materials as skeleton, consists of a helical coil of wire with the lead from one end returning through the centre of the coil to the other end. The Rogowski coil with a winding of uniform density wraps around the current-carrying conductor when measuring the current value. The schematic of a Rogowski coil is illustrated in Fig. 1. The shape of skeleton is toroidal and the shape of section can be rectangle or circular. According to Ampere circuital theorem, the relationship between the current in line $I(t)$ and magnetic flux density that pass through turn area is

$$
B_{i}=\mu_{0} H_{i}=\frac{\mu_{0} I(t)}{2 \pi r}
$$

where $r$ is the distance between centerline of a coil and centerline of the current wire, and $H_{i}$ is magnetic field intensity passing through the coil per turn.

When the area of the coil per turn $S$ is small, the relationship between the induced voltage $e(t)$ and the current $I(t)$ is

$$
e(t)=-n \frac{d \phi}{d t}=-n S \frac{d B_{i}}{d t} \quad \frac{\mu_{0} n S}{2 \pi r} \frac{d I(t)}{d t}
$$

where $n$ is number of turns in a Rogowski coil.

Thus it can be seen that the output voltage of a Rogowski coil is proportional to the time derivative of the busbar current. The measurement of busbar current can be implemented through integrating processing after measuring the output voltage of the Rogowski coil.

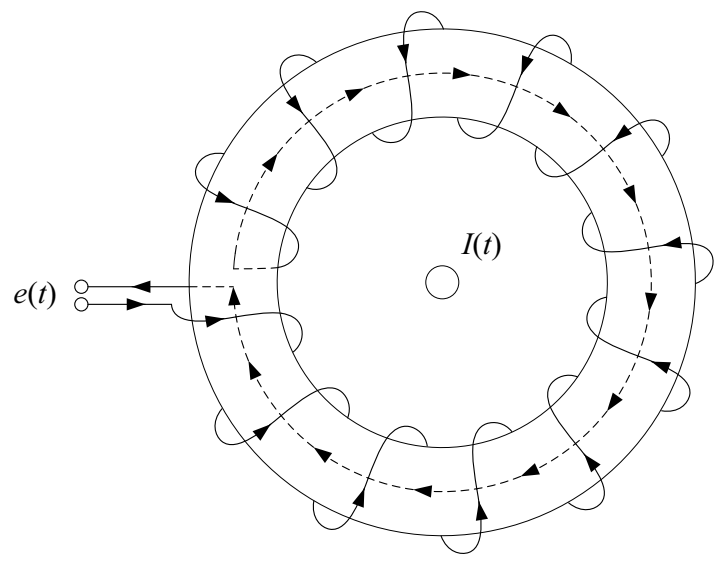

Figure 1. Schematic of sensor section in a Rogowski coil

Sources of Error Created by Proximity Magnetic Field. Many factors influencing the error of Rogowski coils are controllable after many years research. However, the research on proximity magnetic field influence in error of active electronic current transformers rarely gets enough attention.

The sensor of a Rogowski coil indirect measures the current through measuring the induced voltage from variable magnetic field created by the current. However, the measuring objects at the environment of the substation site tend to be three-phase current in simultaneous operation. The magnetic field created by proximal current busbar becomes disturbance magnetic field, which passes through coil section of the Rogowski coil with magnetic field created by measured current busbar and induces corresponding disturbance voltage. The disturbance voltage is superimposed on ideal measurement signals, which produces error to the measurement result. This kind of disturbance magnetic field influences active ECTs especially medium-voltage active ECTs greatly, and is a significant reason that active ECTs exhibit excessive errors at substation sites.

According to Eq. 2, the output voltage of a Rogowski coil is proportional to the time derivative of the busbar current. When the external disturbance magnetic field reaching the coil, the values of magnetic flux density of all coils $B_{i}$ are no longer equivalent. Proceeding time integration to both side of Eq. 2, the busbar current can be expressed as follows:

$$
I=-\frac{2 \pi r}{\underline{\mu}_{0} n S} \int e(t) d t \quad \frac{2 \pi r}{\mu_{0} n S}\left(S \sum_{i=1}^{n} B_{i}\right) \quad \frac{2 \pi r}{\mu_{0} n} \sum_{i=1}^{n} B_{i}
$$

And the Ampere circuital theorem is still valid even having the external disturbance magnetic field. Hence, the Ampere circuital theorem can be correspondingly expressed as follows:

$$
I=\frac{1}{\mu_{0}} \int_{L} \stackrel{r}{B} d l
$$

Comparing Eq. 3 with Eq. 4, the algorithm principle of a Rogowski coil measuring current is that the line integral of the magnetic flux density around the coil skeleton circle in Ampere circuital theorem is equivalent to the average value of magnetic flux density of all coils multiplying by the perimeter of coil skeleton. The two are completely equal when the coils are symmetric even and the number of turns $\mathrm{n}$ approaches infinity. However, the number of turns is limited and the winding is uneven in reality. The distinction between the two is the origin of measurement error of Rogowski coils. In practical application, some uneven air gap tends to exist at outgoing line side. The PCB Rogowski coils are symmetric even while the number of turns is less leading to winding discontinuity. Both designs can bring error. The error can be eliminated through calibration in the ideal situation but can also be uncertainty error at substation sites with outside disturbance magnetic field.

\section{Analysis of Error Influence of Proximity Effect of Active Electronic Current Transformers}

With respect to active ECTs, different environmental settings around transformers can influence magnetic field distribution and consequently influence error. This section of this paper analyzes the impact of a proximity magnetic 
field using electromagnetic field analysis simulations. The effect degrees of proximity magnetic field in all situations are analyzed and some suggestions and measures to reduce the impact are given.

Three-phase Proximity Magnetic Field Simulation with Invariable Distance. Three-phase active ECTs are in simultaneous operation at intelligent substations. The magnetic field created by the adjacent phase current is disturbance magnetic field to ECT at its own phase. In this condition, the environment of active ECTs is nonideal. The magnetic field distribution of its own phase is changed leading to measurement accuracy of the ECT influenced to a certain degree, especially at special situation like three-phase imbalance.

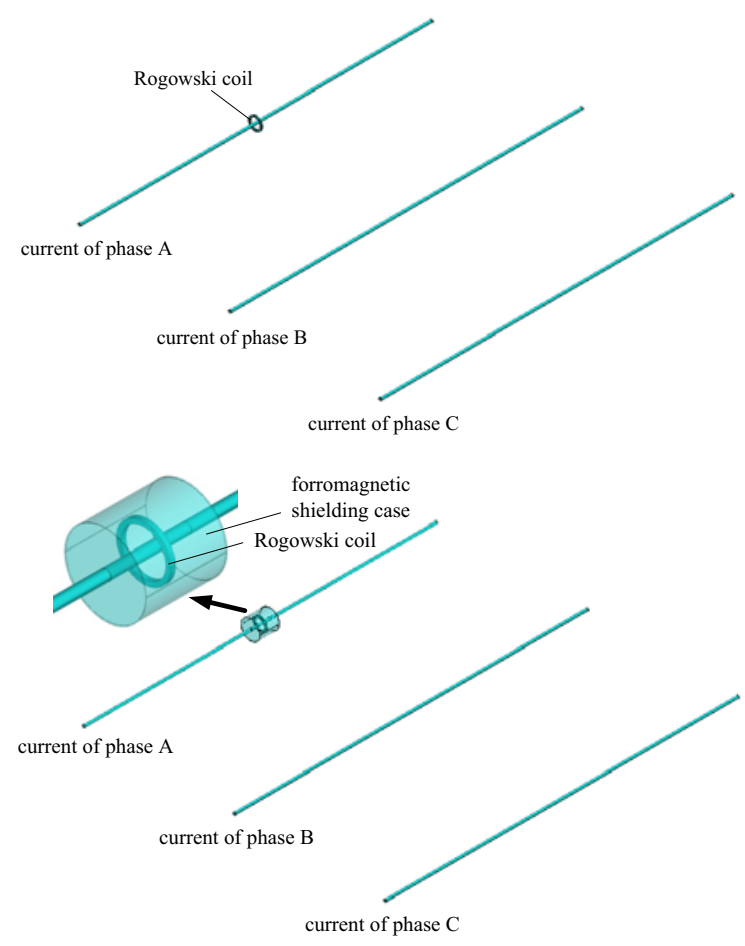

(a)(b)

Figure 2. Model of simulation of (a) three-phase proximity magnetic field (b) three-phase proximity magnetic field with ferro-magnetic shield

Taking $110 \mathrm{kV}$ high voltage line as an example, the model of three-phase proximity magnetic field $3 \mathrm{D}$ simulation is built, which is illustrated in Fig. 2(a). Considering the magnetic field created by current of phase $A$ and phase $C$ are counteracted at the position of phase $B$, this paper aims at research on current of phase $B$ and phase $\mathrm{C}$ influencing an active ECT of phase $\mathrm{A}$. The simulation applies SOLID97 3-D magnetic solid elements and INFIN111 3-D infinite solid elements. The Rogowski coil specific dimensions of phase A are modelled as product dimensions: the inner diameter of Rogowski coils is $140 \mathrm{~mm}$, and the external diameter is $170 \mathrm{~mm}$. The thickness of Rogowski coil is $15 \mathrm{~mm}$, which means cross-section of the coil is a square. The radius of current busbar through the coil is $17 \mathrm{~mm}$, and the electric current density is set as $1.2 \mathrm{~A} / \mathrm{mm}^{2}$. The total length of current busbar is set as double height of ECTs $4.68 \mathrm{~m}$, which can be regard as infinite length and straight line comparing with the coil dimensions. Distance between proximity phases is set as $2 \mathrm{~m}$ according to the requirement of 110 $\mathrm{kV}$ intelligent substations. The Rogowski coil and three current busbar are placed in the air, and it can be considered that nearby devices are infinitely far apart from them (i.e., other devices will not create impacts). In postprocessing, the magnetic flux density in the position of diameter being equal to $155 \mathrm{~mm}$ (mean value of inner diameter and external diameter) integrate along the located circle. Compared with current set value, the theoretical errors of calculated current value after integral calculation in different conditions can be obtained.

In the industry, some active ECTs manufacturers applied ferro-magnetic shield around the Rogowski coil sensors in their active ECT products. Considering ferro-magnetic shield being able to decrease the impact of ambient magnetic field, this paper compares simulation of three-phase proximity magnetic field with ferro-magnetic shield against simulation of three-phase proximity magnetic field without ferro-magnetic shield to get contrastive analysis of error influence quantities. The model of ferro-magnetic shield is a cylinder with the external diameter being $240 \mathrm{~mm}$, the inner diameter being $236 \mathrm{~mm}$ and length being $240 \mathrm{~mm}$. The relative permeability of ferro-magnetic shield $\mu$ is 400 , the same as the relative permeability of cast iron. The model of simulation of three-phase proximity magnetic field with ferro-magnetic shield is illustrated in Fig. 2(b).

Fig. 3(a) illustrates a magnetic flux density distribution pattern of the Rogowski coil in the ideal condition of the phase A current being $100 \% I_{N}$ without the impact of disturbance magnetic field from proximity phases. By contrast, Fig. 3(b) illustrates a magnetic flux density distribution pattern of the Rogowski coil in the condition of three-phase current all being $100 \% I_{N}$. And Fig. 3(c) illustrates a magnetic flux density distribution pattern of the Rogowski coil in the same condition as Fig. 3(b) but with ferro-magnetic shield. From Fig. 3(a) to Fig. 3(c), it can be concluded that applying ferro-magnetic shield around the Rogowski coil can reduce the disturbance from proximity magnetic field and make the measured magnetic field distribution closer to ideal situations.

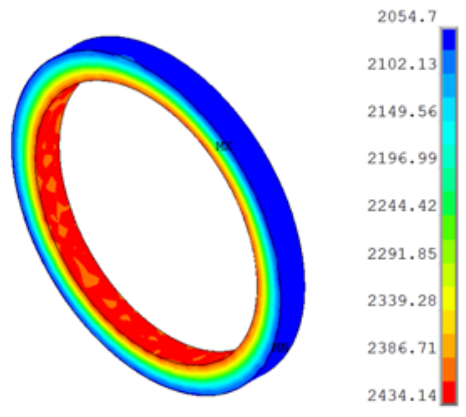



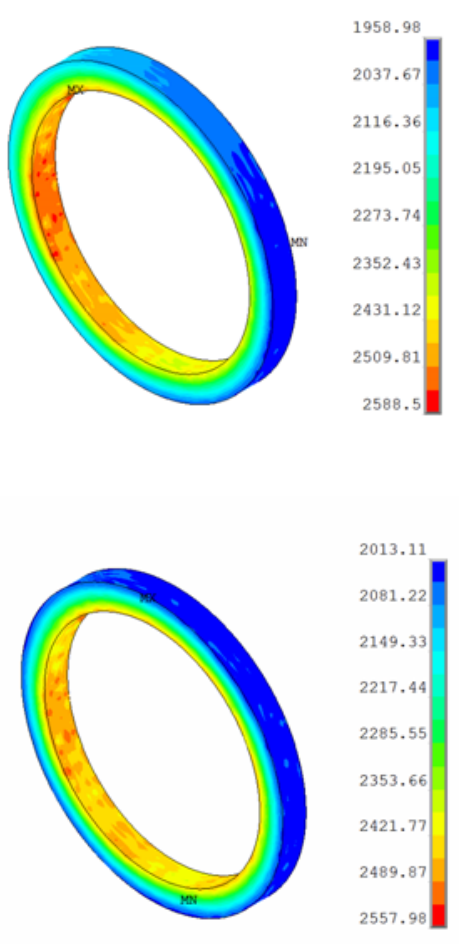

(a) (c) (b)

Figure 3. Magnetic flux density distribution pattern of the Rogowski coil (a) in the ideal condition (b) with proximity magnetic field disturbance (c) with ferro-magnetic shield

Based on IEC standard [12], error of ECTs is requested within limits of error at four percentages of rated current: $5 \% I_{N}, 20 \% I_{N}, 100 \% I_{N}$ and $120 \% I_{N}$. Testing department conducts the accuracy test and error calibration to ECTs also at the four percentages of rated current. Hence, this paper considers the situation of phase $A$ at four percentages of rated current influenced by three-phase proximity magnetic field. The current values of phase $\mathrm{B}$ and phase $\mathrm{C}$ stay the same all along the simulations and the variation range is from $5 \% I_{N}$ to $120 \% I_{N}$. Current error of phase A in the conditions of not applying ferro-magnetic shield and applying ferro-magnetic shield are demonstrated in Fig. 4(a) and Fig. 4(b), respectively. The current error of phase A is 0 when applying ferro-magnetic shield and the current of phase $\mathrm{B}$ and phase $\mathrm{C}$ are at $5 \% I_{N}$. So the simulation data of phase $\mathrm{B}$ and phase $\mathrm{C}$ at $5 \% I_{N}$ is not shown in Fig. 4(b).
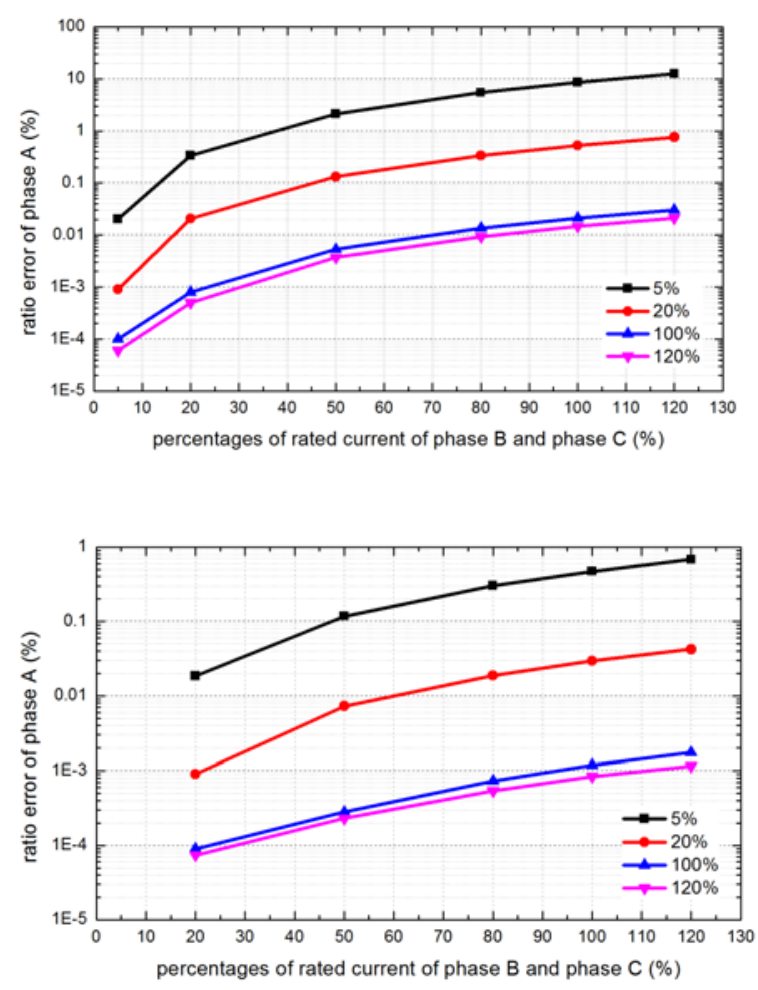

(a) (b)

Figure 4. The Rogowski coil error of phase A (a) without ferro-magnetic (b) shield with ferro-magnetic shield

With regard to 0.2 class ECTs, the limits of ratio error at $5 \% I_{N}, 20 \% I_{N}, 100 \% I_{N}$ and $120 \% I_{N}$ are $0.75 \%, 0.35 \%$, $0.2 \%$ and $0.2 \%$, respectively. Only the maximum ratio error caused by a three-phase proximity magnetic field being maintained better than $0.075 \%, 0.035 \%, 0.02 \%$ and $0.02 \%$ correspondingly can the influence be considered negligible. The simulation results indicate that the impact of the three-phase proximity magnetic field increases with the current value of phase $\mathrm{B}$ and phase $\mathrm{C}$ increasing. Under the same conditions, error of active ECTs caused by a three-phase proximity magnetic field reduces dramatically after adding a ferro-magnetic shield. Without ferro-magnetic shield, the impacts of current of phase A at $100 \% I_{N}$ and $120 \% I_{N}$ influenced by three-phase proximity magnetic field can be considered negligible. While the impacts at $20 \% I_{N}$ and $5 \% I_{N}$ influenced by three-phase proximity magnetic field make active ECTs exhibit excessive errors seriously. With ferro-magnetic shield, the impacts of current of phase A at $20 \% I_{N}$, $100 \% I_{N}$ and $120 \% I_{N}$ influenced by three-phase proximity magnetic field can be considered negligible. And the impacts at $5 \% I_{N}$ influenced by three-phase proximity magnetic field still make excessive errors phenomenon possibly.

Combining the results of Fig. 4(a) and Fig. 4(b), the following conclusion can be reached.

1) Under the condition of three-phase current equilibrium, active ECTs are less influenced by three-phase proximity magnetic field. 
2) Under the condition of minor three-phase current imbalance, the influence by three-phase proximity magnetic field to active ECTs should not be ignored. Applying ferro-magnetic shield outside active ECTs can decrease the influence and guarantee the accuracy of measurement.

3) Under the condition of major three-phase current imbalance (e.g. there are $5 \% I_{N}$ and $120 \% I_{N}$ in three phases simultaneously), the disturbance of three-phase proximity magnetic field is not able to eliminate even though applying ferro-magnetic shield.

Three-phase Proximity Magnetic Field Simulation with Variable Distance. Distance between proximity phases of $110 \mathrm{kV}$ and above voltage classes is small, which is $2 \mathrm{~m}$ generally. The possibility of corresponding current in the condition of major three-phase current imbalance is also small. Hence, the impact of three-phase proximity magnetic field is relative small. With regard to $10 \mathrm{kV}$ voltage class, the corresponding ECTs are installed in power distribution cabinets with small distance between phases. In this condition, the disturbance of three-phase proximity magnetic field can change magnetic field distribution much more and creates worse impacts. Active ECTs probably exhibit excessive errors even though the three-phase current is balanced. Changing the distance between phases as a variable in the model of Fig. 2(a) and Fig. 2(b), and the distance is chosen from 0.2 to $2.0 \mathrm{~m}$. The different relative permeability of ferro-magnetic shield $\mu$ is chosen from 400 to 1000 in the new simulation. The three-phase current is set as $100 \% I_{N}$ under the condition of three-phase current equilibrium to simulate active medium-voltage ECTs influenced by three-phase proximity magnetic field.
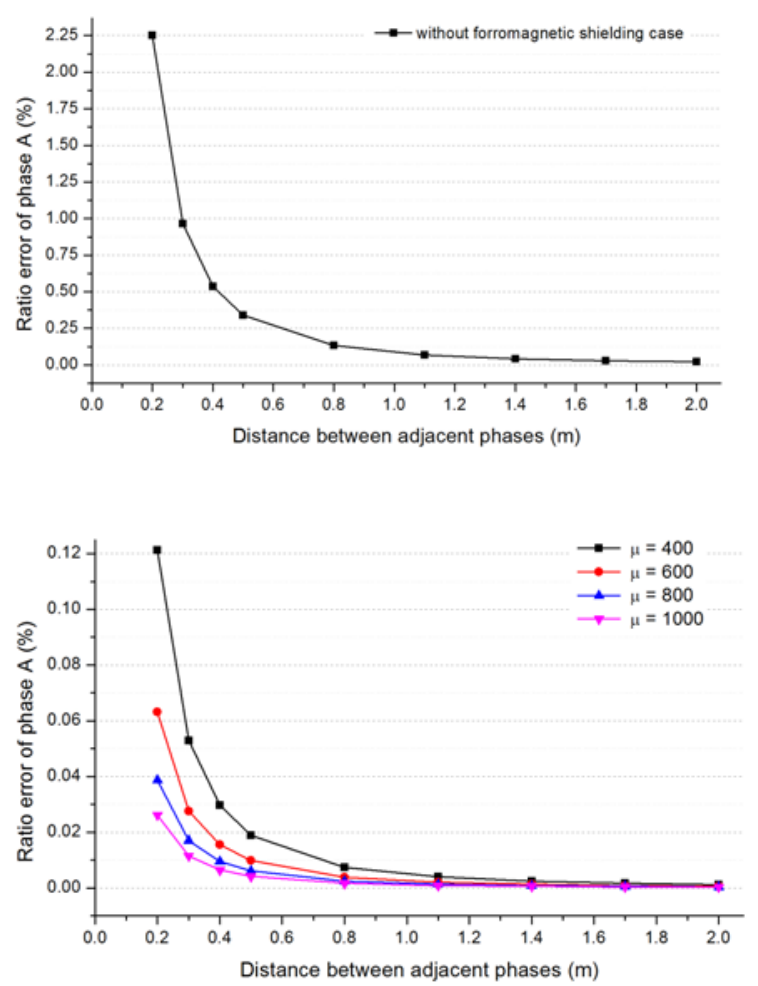

(a)(b)

Figure 5.. The error of active medium-voltage ECTs influenced by three-phase proximity magnetic field (a) without ferro-magnetic shield (b) with ferro-magnetic shield

The simulation results are shown in Fig. 5(a) and Fig. 5(b). The impacts of three-phase proximity magnetic field increase with the decrease of distance between phases. To active medium-voltage ECTs without ferro-magnetic shield, the ratio error exceeds the limit of error $0.2 \%$ when distance between phases reduces to approximate 0.7 $\mathrm{m}$. At this distance point, the impacts of three-phase proximity magnetic field have already made active ECTs exhibit excessive errors. To active medium-voltage ECTs with ferro-magnetic shield, the impacts of three-phase proximity magnetic field decrease with the increasement of relative permeability of ferro-magnetic shield. But under the condition where relative permeability of ferro-magnetic shield $\mu \leq 1000$ and the distance between phases is $0.2 \mathrm{~m}$, the impacts of three-phase proximity magnetic field still cannot be neglected. Combining the simulation data, conclusions can be reached as follows.

1) Under the condition where relative permeability of ferro-magnetic shield $\mu \geq 400$ and the distance between three-phases current lines is equal or greater than $0.5 \mathrm{~m}$, the maximum ratio error of transformers that is caused by three-phase proximity magnetic field can be maintained at a value that is better than $+0.02 \%$.

2) Under the condition where relative permeability of ferro-magnetic shield $\mu \geq 800$ and the distance between three-phases current lines is equal or greater than $0.3 \mathrm{~m}$, the maximum ratio error of transformers that is caused by three-phase proximity magnetic field can be maintained at a value that is better than $+0.02 \%$ as well.

Combining all the results of three-phase proximity magnetic field simulations, three suggestions are given as follows.

1) With regard to active ECTs with Rogowski coil, it is suggested that manufacturers use ferro-magnetic shield to reduce disturbance magnetic field. The relative permeability of ferro-magnetic shield is suggested around 400 for active high-voltage ECTs while it is suggested above 1000 for active medium-voltage ECTs.

2) Under the condition of major three-phase current imbalance (e.g. the railway grid has major three-phase current imbalance because of articulated phase in its operation mode), the additional error of active ECTs should be taken into consideration.

3) The impacts of three-phase proximity magnetic field increase with the decrease of distance between phases. It is suggested to guarantee the distance between phases more than $0.3 \mathrm{~m}$ in power distribution cabinet for medium-voltage ECTs.

\section{Conclusion}

Error of active ECTs with Rogowski coil at substation site is influenced by proximity magnetic field. For high-voltage transformers, the influence of proximity magnetic field mainly occurs under the condition of three-phase current imbalance; for medium-voltage 
transformers, the influence of proximity magnetic field can still occur under the condition of three-phase current equilibrium. The influence is a significant reason that triggers off a disparity between the error characteristics of active ECTs in the laboratory and at the substation site. Applying ferro-magnetic shield with high relative permeability in the design structure of active ECTs with Rogowski coil and guaranteeing distance between three-phase current lines can reduce the influence of proximity magnetic field.

\section{References}

1. B. Djokic, E. So, IEEE Trans. Instrum. Meas., 54 (2005) pp. 479-482

2. A. Ehsan, M. Richard, IEEE Trans. Instrum. Meas., 56 (2007) pp. 753-759

3. G. Crotti, D. Gallo, D. Giordano, C. Landi and M. Luiso, IEEE Trans. Instrum. Meas., 63 (2014) pp. 2349-2357

4. X. Xiao, Y. Xu and Z. Dong, Sensors, 15 (2015) pp. $7125-7135$

5. I. A. Metwally, IEEE SENSORS J., 13 (2013) pp. 538-547

6. I. A. Metwally, IEEE Trans. Instrum. Meas., 59 (2010) pp. 353-360

7. Y. Liu, F. Lin, Q. Zhang, and H. Zhong, IEEE SENSORS J., 11 (2011) 123-130

8. E. Suomalainen, J. K. Hällström, IEEE Trans. Instrum. Meas., 58 (2009) pp. 1054-1058

9. I. A. Metwally, IEEE Trans. Instrum. Meas., 62 (2013) pp. 2303-2313

10. P. Orr, P. Niewczas, C. Booth, G. Fusiek, A. Dysko, F. Kawano, T. Nishida and P. Beaumont, IEEE SENSORS J., 13 (2013) pp. 2053-2054

11. M. H. Samimi, A. Mahari, M. A. Farahnakian, and H. Mohseni, IEEE SENSORS J., 15 (2015) pp. 651-658

12. Instrument Transformers-Part 8: Electronic Current Transformers, document IEC 60044-8, Geneva, Switzerland, (2002) 